\title{
OPERATORS WITH SINGULAR CONTINUOUS SPECTRUM, V. SPARSE POTENTIALS
}

\author{
B. SIMON $^{1}$ AND G. STOLZ ${ }^{2}$
}

\begin{abstract}
By presenting simple theorems for the absence of positive eigenvalues for certain one-dimensional Schrödinger operators, we are able to construct explicit potentials which yield purely singular continuous spectrum.
\end{abstract}

\section{$\S 1$. Introduction}

This is one of a series of papers (see $[2,11,3,7,1,13,4,6,12]$ ) that discusses singular continuous spectrum in families of concrete operators. Our goal here is to provide examples of half-line Schrödinger operators which have singular continuous spectrum $[0, \infty)$ for all boundary conditions at $x=0$. In fact, singular continuity of the spectrum will be preserved under arbitrary compactly supported perturbations for these examples. This is of interest because it provides totally explicit operators without a need to appeal to generic values of parameters and because it is a concrete example of the fact that while dense point spectrum is always unstable under rank one perturbations [3], singular continuous spectrum may be stable.

To be totally explicit, it will follow from our discussion below that if $x_{n}=e^{2 n^{3 / 2}}$, $n=1,2, \ldots$ and

$$
V(x)= \begin{cases}n & \left|x-x_{n}\right|<\frac{1}{2} \\ 0 & \text { otherwise }\end{cases}
$$

then for any boundary condition at $0, H \equiv-\frac{d^{2}}{d x^{2}}+V(x)$ on $[0, \infty)$ has $\sigma(H)=\sigma_{\mathrm{sc}}(H)=$ $[0, \infty)$ and $\sigma_{\mathrm{ac}}(H)=\emptyset$. Depending on the boundary condition, $\sigma_{\mathrm{pp}}(H)$ is either $\emptyset$ or a single negative eigenvalue. And the whole-line problem, defined by symmetric extension of $V$, has $\sigma(H)=\sigma_{\mathrm{sc}}(H)=[0, \infty), \sigma_{\mathrm{ac}}(H)=\sigma_{\mathrm{pp}}(H)=\emptyset$.

Our examples here will be sparse, that is, they will be "mainly" zero. Our main technical result, in Section 2, will show that sparse potentials have no point spectrum in $[0, \infty)$ for any boundary condition. The proof will be very elementary. The examples of singular continuous spectrum in Section 3 will then come by combining the results of Sections 2 and 3 with the theorems of Simon-Spencer [14] and Stolz [15] on the absence of absolutely continuous spectrum for certain potentials.

\footnotetext{
1 Division of Physics, Mathematics and Astronomy, California Institute of Technology, 253-37, Pasadena, CA 91125. This material is based upon work supported by the National Science Foundation under Grant No. DMS-9101715. The Government has certain rights in this material.

2 Department of Mathematics, University of Alabama, Birmingham, AL 35294.

To appear in Proc. Amer. Math. Soc.
} 
In Section 4 we give two other situations where our ideas apply: If $V$ is mainly periodic instead of mainly zero, then one gets singular continuity in the spectral bands of the underlying periodic potential. It is also easy to extend our results to Jacobi matrices.

It has not escaped our notice that Pearson's examples [10] are sparse and, indeed, our Section 2 implies the absence of point spectrum in his examples (for any boundary condition!). However, we know of no way yet to prove the absence of absolutely continuous spectrum in his examples other than the one he uses. At least, combining our argument with the one of Pearson shows that Pearson's examples also work on the whole line, a fact not noted in [10].

We do note, however, that Last and Simon (work in progress) have another way to construct potentials which decay at infinity and have purely singular continuous spectrum and by [11], Baire generic $V$ 's vanishing at infinity have singular continuous spectrum on $[0, \infty)$.

A result of the type discussed in this paper has been stated before in [5], but with no proof. After we completed this work, we learned from [9] of some apparently unpublished results of Gordon on Jacobi matrices with sparse potentials with a similar flavor to what we do.

G.S. would like to thank M. Aschbacher and C. Peck for the hospitality of Caltech where most of this work was done.

\section{§2. Absence of Point Spectrum}

Let $V(x)$ be a measurable function on $[0, \infty)$ which is $L^{1}$ on any interval $[0, R)$. For $y \geq 0$, one can look at solutions of the differential equation

$$
\begin{gathered}
-u^{\prime \prime}(x)+V(x) u(x)=E u(x) \\
u(y)=a \quad u^{\prime}(y)=b .
\end{gathered}
$$

If $\Phi(x, y, E ; V)$ is the solution with $\left(\begin{array}{l}a \\ b\end{array}\right)=\left(\begin{array}{l}1 \\ 0\end{array}\right)$ and $\Psi(x, y, E ; V)$ with $\left(\begin{array}{l}a \\ b\end{array}\right)=\left(\begin{array}{l}0 \\ 1\end{array}\right)$, then the $2 \times 2$ matrix

$$
M(x, y, E ; v)=\left(\begin{array}{cc}
\Phi(x, y, E ; V) & \Psi(x, y, E ; V) \\
\Phi^{\prime}(x, y, E ; V) & \Psi^{\prime}(x, y, E ; V)
\end{array}\right)
$$

is called the transfer or fundamental matrix. Solutions of $-u^{\prime \prime}+V u=E u$ obey

$$
\left(\begin{array}{c}
u(x) \\
u^{\prime}(x)
\end{array}\right)=M(x, y, E ; V)\left(\begin{array}{c}
u(y) \\
u^{\prime}(y)
\end{array}\right) .
$$

and

$$
M(x, y) M(y, z)=M(x, z)
$$

Constancy of the Wronskian implies that

$$
\operatorname{det}(M)=1
$$


Theorem 2.1. If $V$ is bounded from below and $M$ obeys

$$
\int_{0}^{\infty} \frac{d x}{\|M(x, 0, E ; V)\|^{2}}=\infty
$$

for some $E$, then $-u^{\prime \prime}+V u=E u$ has no solution $u \in L^{2}(0, \infty)$.

Proof. Since $M$ is unimodular ((1)) and $2 \times 2$, we have

$$
\left\|M^{-1}\right\|=\|M\|
$$

By (1),

$$
\left(\begin{array}{c}
u(0) \\
u^{\prime}(0)
\end{array}\right)=M(x, 0)^{-1}\left(\begin{array}{c}
u(x) \\
u^{\prime}(x)
\end{array}\right) .
$$

So, by (5)

$$
\left\|\left(\begin{array}{c}
u(x) \\
u^{\prime}(x)
\end{array}\right)\right\| \geq\left\|\left(\begin{array}{c}
u(0) \\
u^{\prime}(0)
\end{array}\right)\right\| /\|M(x, 0)\| .
$$

Thus, (4) implies that $\left\|\left(\begin{array}{c}u \\ u^{\prime}\end{array}\right)\right\| \notin L^{2}$. Then also $u \notin L^{2}$.

Suppose, on the contrary, that $u \in L^{2}$. Differentiating $u u^{\prime}$, we get

$$
\begin{aligned}
u(x) u^{\prime}(x) & =u(0) u^{\prime}(0)+\int_{0}^{x}\left(u^{\prime 2}+u^{2}(V-E)\right) d t \\
& \geq u(0) u^{\prime}(0)+\int_{0}^{x}\left(u^{\prime 2}-C u^{2}\right) d t,
\end{aligned}
$$

where semiboundedness of $V$ was used. If $u \in L^{2}$, but $u^{\prime} \notin L^{2}$, this yields $\frac{1}{2}\left(u^{2}\right)^{\prime}(x)=$ $u(x) u^{\prime}(x) \rightarrow \infty$ as $x \rightarrow \infty$. This would imply the contradiction $u^{2}(x) \rightarrow \infty$.

We are going to apply this to what we'll call sparse potentials.

Definition. A sparse potential is a function $V(x)$ on $[0, \infty)$ for which there exist $x_{n+1}>$ $x_{n} \rightarrow \infty, \alpha_{n}>0$ and $h_{n}<\infty$ so

(i) $\frac{x_{n+1}-x_{n}}{\alpha_{n}+\alpha_{n+1}+1} \rightarrow \infty$,

(ii) $|V(x)| \leq h_{n}$ if $\left|x-x_{n}\right| \leq \alpha_{n}, n=1,2, \ldots$,

(iii) $\mid V(x)=0$ otherwise.

By (i), (iii), sparse potentials are zero "most of the time." We define $L_{n}=x_{n+1}-x_{n}-$ $\alpha_{n}-\alpha_{n+1}$. 
Theorem 2.2. Let $V$ be a sparse potential. Suppose that

$$
L_{n} \geq \exp \left(4 Q_{n}\right)
$$

for all large $n$ where

$$
Q_{n} \equiv n \ln n+\sum_{j=1}^{n} \alpha_{j}\left(h_{j}+\ln j\right)
$$

Then $-u^{\prime \prime}+V u=E u$ has no solutions with $u \in L^{2}$ for any $E>0$.

Proof. $M^{\prime}(x)=A(x) M(x)$ where

$$
A(x)=\left(\begin{array}{cc}
0 & 1 \\
-1 & E-V(x)
\end{array}\right)
$$

so $\|A(x)\|=|V(x)|+E+1 \leq h_{n}+E+1$ on $\left(x_{n}-\alpha_{n}, x_{n}+\alpha_{n}\right)$ and

$$
\left\|M\left(x_{n}+\alpha_{n}, x_{n}-\alpha_{n}\right)\right\| \leq e^{2\left(h_{n}+E+1\right) \alpha_{n}} .
$$

On the other hand, the transfer matrix when $V=0$ and $E=k^{2}>0$ is

$$
M_{0}(x, y)=\left(\begin{array}{cc}
\cos k(x-y) & k^{-1} \sin k(x-y) \\
-k \sin k(x-y) & \cos k(x-y)
\end{array}\right)
$$

and has norm bounded by $\max \left(k, k^{-1}\right)$.

Using (2), it follows that for $y \in\left(x_{n}+\alpha_{n}, x_{n+1}-\alpha_{n+1}\right)$, we have

$$
\|M(y, 0)\| \leq\left[\max \left(k, k^{-1}\right)\right]^{n+1} \exp \left(\sum_{j=1}^{n} 2 \alpha_{j}\left(h_{j}+E+1\right)\right) \leq \exp \left(2 Q_{n}\right)
$$

for $n$ large (and any fixed $E$ ). Thus,

$$
\int_{x_{n}+\alpha_{n}}^{x_{n+1}-\alpha_{n+1}} \frac{d y}{\|M(y, 0)\|^{2}} \geq \frac{L_{n}}{\exp \left(4 Q_{n}\right)} \geq 1
$$

by hypothesis. Thus, (4) holds and there are no $L^{2}$ solutions.

Example 1. $h_{n}=n, \alpha_{n}=\frac{1}{2} ;(6)$ requires $L_{n} \geq \exp \left((1+\epsilon) n^{2}\right)$.

Example 2. $h_{n}=c, \alpha_{n}=n$; (6) requires $L_{n} \geq \exp \left((1+\epsilon) n^{2}\right)$ again.

In the case of constant high barriers, like in Example 1, one actually has the following result, which gives improved estimates 
Theorem 2.3. Let $V$ be a sparse potential with

$$
V(x)=h_{n} \quad \text { if }\left|x-x_{n}\right| \leq a_{n}, n=1,2, \ldots,
$$

$h_{n} \rightarrow \infty$ and

$$
L_{n} \geq n^{\delta n}\left(\prod_{j=1}^{n} h_{j}\right) \exp \left(4 \sum_{j=1}^{n} \alpha_{j} \sqrt{h_{j}}\right)
$$

for some $\delta>0$. Then $-u^{\prime \prime}+V u=E u$ has no solutions with $u \in L^{2}$ for any $E>0$.

Looking at Example 1 again, we see that (7) only requires $L_{n} \geq \exp \left((1+\epsilon) n^{3 / 2}\right)$.

Proof. We follow the proof of Theorem 2.2, but for $n$ with $h_{n}>E+1$ and $x, y \in\left[x_{n}-\right.$ $\left.\alpha_{n}, x_{n}+\alpha_{n}\right]$, replace $M$ by the modified transfer matrix

$$
\tilde{M}_{n}(x, y)=\left(\begin{array}{cc}
\sqrt{h_{n}-E} \Phi & \sqrt{h_{n}-E} \Psi \\
\Phi^{\prime} & \Psi^{\prime}
\end{array}\right) .
$$

We have

$$
\tilde{M}_{n}^{\prime}=\sqrt{h_{n}-E}\left(\begin{array}{ll}
0 & 1 \\
1 & 0
\end{array}\right) \tilde{M}_{n}
$$

and therefore,

$$
\left\|\tilde{M}_{n}\left(x_{n}+\alpha_{n}, x_{n}-\alpha_{n}\right)\right\| \leq \exp \left(2 \alpha_{n} \sqrt{h_{n}-E}\right)
$$

Since

$$
M\left(x_{n}+\alpha_{n}, x_{n}-\alpha_{n}\right)=\left(\begin{array}{cc}
\left(h_{n}-E\right)^{-1 / 2} & 0 \\
0 & 1
\end{array}\right) \tilde{M}_{n}\left(x_{n}+\alpha_{n}, x_{n}-\alpha_{n}\right)\left(\begin{array}{cc}
\left(h_{n}-E\right)^{1 / 2} & 0 \\
0 & 1
\end{array}\right)
$$

we get

$$
\begin{aligned}
\left\|M\left(x_{n}+\alpha_{n}, x_{n}-\alpha_{n}\right)\right\| & \leq \max \left(\left(h_{n}-E\right)^{1 / 2},\left(h_{n}-E\right)^{1 / 2}\right) \exp \left(2 \alpha_{n} \sqrt{h_{n}-E}\right) \\
& \leq \sqrt{h_{n}} \exp \left(2 \alpha_{n} \sqrt{h_{n}}\right) .
\end{aligned}
$$

Now the proof is completed as before.

\section{$\S 3$. Examples with Singular Continuous Spectrum}

As stated in the introduction, the idea behind these examples is to use [14] and [15] to eliminate a.c. spectrum and Theorems 2.2 and 2.3 to eliminate point spectrum. Both results apply with arbitrary bounded condition at $x=0$. Non-existence of a.c. spectrum is also stable under a compactly supported perturbation of $V$ (a trace class perturbation of the resolvent, e.g., [4]). Obviously, the same is true for non-existence of square-integrable solutions.

Therefore, we get examples with purely singular continuous spectrum for all boundary conditions and under arbitrary local perturbations. Whole-line problems differ from the direct sum of two half-line problems only by adding boundary conditions at 0 , a finite rank perturbation. Moreover, lack of $L^{2}$ solutions on either half-line clearly implies no $L^{2}$ solutions on the whole line. This shows that we also get whole-line examples of purely singular continuous spectrum.

The statements on $\sigma_{\mathrm{pp}}$ in $(-\infty, 0]$ made in the introduction follow from elementary convexity considerations for solutions. 
Example 3. Let $x_{n}=e^{2 n^{2 / 3}}$ and

$$
V(x)= \begin{cases}n & \text { if }\left|x-x_{n}\right|<\frac{1}{2}, n=1,2, \ldots \\ 0 & \text { otherwise }\end{cases}
$$

Then $-\frac{d^{2}}{d x^{2}}+V(x)$ on $L^{2}(0, \infty)$ has purely singular continuous spectrum in $(0, \infty)$ for any boundary condition at $x=0$. For [14] says there is no a.c. spectrum and Theorem 2.3 says no point spectrum.

Example 4. Here we construct bounded potentials with purely singular continuous spectrum in $(0, \infty)$. Let $W(x)$ be the random potential given by a random constant $c_{n}$ in each interval $(n-1, n), n=1,2 \ldots$, where the $c_{n}$ are independent and each uniformly distributed in $[0,1]$. Let

$$
V(x)= \begin{cases}W\left(x+\frac{n(n-1)}{2}-x_{n}\right), & x_{n} \leq x \leq x_{n}+n \\ 0 & \text { otherwise }\end{cases}
$$

where $x_{n}=e^{2 n^{2}}$. Essentially, we've broken $W(x)$ into pieces of size $1,2, \ldots$ and placed them at the points $x_{1}, x_{2} \ldots$ Then for almost all choices of the random potential, $-\frac{d^{2}}{d x^{2}}+$ $V(x)$ on $L^{2}(0, \infty)$ has purely singular continuous spectrum for all boundary conditions at $x=0$.

By Theorem 2.2 there is no point spectrum. To prove absence of a.c. spectrum, let $\chi$ be the characteristic function on $[0,1]$ and

$$
q_{L}(x)=\sum_{n=-\infty}^{\infty} \chi(x-n L)
$$

If $E_{0}=k_{0}^{2}>0$ is such that $\frac{k_{0}}{2 \pi}$ is not rational, then Theorem 3.4 of [8] guarantees the existence of an integer $L=L\left(E_{0}\right)$ and $\delta>0$ such that $\left(E_{0}-\delta, E_{0}+\delta\right)$ is contained in a spectral gap of $-\frac{d^{2}}{d x^{2}}+q_{L}$. Almost certainly, there are intervals $I_{n}$ with length tending to $\infty$ such that

$$
\sup _{x \in \cup I_{n}}\left|W(x)-q_{L}(x)\right|<\frac{\delta}{2} .
$$

By construction, the same holds for $V$. By Theorem 1 of [15], we have $\sigma_{\text {ac }}\left(-\frac{d^{2}}{d x^{2}}+V\right) \cap$ $\left(E_{0}-\frac{\delta}{2}, E_{0}+\frac{\delta}{2}\right)=\emptyset$ with probability one. A compactness argument and the fact that the countable set $\left\{k_{0}^{2}: \frac{k_{0}}{2 \pi}\right.$ rational $\}$ cannot support a.c. spectrum finally show almost sure absence of a.c. spectrum.

Of course, the above construction and argument apply to much more general random potentials $W$. 


\section{$\S 4$. "Mainly" Periodic Potentials and Jacobi Matrices}

In Section 2 the important property of the regions with $V=0$ was that, for a given $E>0$, the norm of the transfer matrix $M_{0}(x, y)$ is uniformly bounded in $x, y$. We are in the same situation if we look at the transfer matrix for $-u^{\prime \prime}+V_{0} u=E u$, where $V_{0}$ is periodic and $E$ is an interior point of one of the stability intervals for $-\frac{d^{2}}{d x^{2}}+V_{0}$. Thus, all our results for sparse potentials have suitable extensions to "mainly" periodic potentials. We illustrate this with

Example 5. Let $V_{0}$ be real and periodic, $x_{n}=e^{2 n^{3 / 2}}$ and

$$
V(x)= \begin{cases}n & \left|x-x_{n}\right|<\frac{1}{2}, n=1,2, \ldots \\ V_{0}(x) & \text { otherwise }\end{cases}
$$

Then $-\frac{d^{2}}{d x^{2}}+V$ on $L^{2}(0, \infty)$ with any boundary condition at $x=0$ has purely singular continuous spectrum in the interior of the stability intervals of $-\frac{d^{2}}{d x^{2}}+V_{0}$.

The above methods can easily be applied to Jacobi matrices $h$ on $\ell^{2}(0, \infty)$ defined by

$$
\begin{aligned}
& (h u)(0)=u(1)+v(0) u(0), \\
& (h u)(x)=u(x-1)+u(x+1)+v(x) u(x), \quad x=1,2, \ldots
\end{aligned}
$$

As an example, we give

Theorem 4.1. Let $h_{n} \rightarrow \infty, x_{n}$ be integers with $x_{n+1}>x_{n} \rightarrow \infty$ and

$$
v(x)= \begin{cases}h_{n} & x=x_{n}, n=1,2, \ldots \\ 0 & \text { otherwise }\end{cases}
$$

Furthermore, let $\ell_{n}=x_{n+1}-x_{n}$ satisfy

$$
\ell_{n} \geq \prod_{j=1}^{n}\left(h_{j}+\ln j\right), \quad n=1,2, \ldots
$$

Then, the Jacobi matrix $h$ is purely singular continuous in $(-2,2)$.

Proof. $\sigma_{\mathrm{ac}}(h)=\emptyset$ follows from $h_{n} \rightarrow \infty$ and [14].

The transfer matrix to solutions of

$$
u(x-1)+u(x+1)+v(x) u(x)=e u(x), \quad x=1,2, \ldots
$$

is given by

$$
M(x)=\prod_{j=1}^{x} T(j)
$$

where

$$
T(j)=\left(\begin{array}{cc}
0 & 1 \\
-1 & e-v(j)
\end{array}\right)
$$


By an analog to Theorem 2.2, it suffices to show that for $e \in(-2,2)$

$$
\sum_{x=1}^{\infty} \frac{1}{\|M(x)\|^{2}}=\infty \text {. }
$$

We have

$$
\left\|T\left(x_{n}\right)\right\| \leq h_{n}+|e|+1
$$

and, diagonalizing $\left(\begin{array}{cc}0 & 1 \\ -1 & e\end{array}\right)$ for $e \in(-2,2)$,

$$
\left\|\prod_{j=x_{n}+1}^{x_{n+1}-1} T(j)\right\| \leq C(e)
$$

uniformly in $n$. Thus,

$$
\|M(x)\| \leq C(e)^{n+1} \prod_{j=1}^{n}\left(h_{j}+|e|+1\right)
$$

for $x \in\left(x_{n}, x_{n+1}\right)$. (9) follows from this and (8).

\section{REFERENCES}

[1] R. del Rio, S. Jitomirskaya, Y. Last, and B. Simon, Operators with singular continuous spectrum, IV. Hausdorff dimension, rank one perturbations, and localization, in preparation.

[2] R. del Rio, S. Jitomirskaya, N. Makarov, and B. Simon, Singular spectrum is generic, Bull. Amer. Math. Soc. 31 (1994), 208-212.

[3] R. del Rio, N. Makarov, and B. Simon, Operators with singular continuous spectrum, II. Rank one operators, Commun. Math. Phys. 165 (1994), 59-67.

[4] R. del Rio, B. Simon, and G. Stolz, Stability of spectral types for Sturm-Liouville operators, Math. Research Lett. 1 (1994), 437-450.

[5] A. Gordon, S. Molchanov, and B. Tsagani, Spectral theory for one-dimensional Schrödinger operators with strongly fluctuating potentials, Funct. Anal. Appl. 25 (1992), 236-238.

[6] A. Hof, O. Knill, and B. Simon, Singular continuous spectrum for palindromic Schrödinger operators, Commun. Math. Phys. (to appear).

[7] S. Jitomirskaya and B. Simon, Operators with singular continuous spectrum, III. Almost periodic Schrödinger operators, Commun. Math. Phys. 165 (1994), 201-205.

[8] W. Kirsch, S. Kotani, and B. Simon, Absence of absolutely continuous spectrum for some onedimensional random but deterministic potentials, Ann. Inst. Henri Poincaré 42 (1985), 383-406.

[9] S. Molchanov, Lectures on the Random Media, Summer School in Probability Theory, Saint-Flour, France, 1992.

[10] D. Pearson, Singular continuous measures in scattering theory, Commun. Math. Phys. 60 (1978), $13-36$.

[11] B. Simon, Operators with singular continuous spectrum, I. General operators, Ann. of Math. 141 (1995), 131-145.

$[12] \longleftarrow L^{p}$ norms of the Borel transform and the decomposition of measures, Proc. Amer. Math. Soc. (to appear).

[13] - Operators with singular continuous spectrum, VI. Graph Laplacians and Laplace-Beltrami operators, Proc. Amer. Math. Soc. (to appear).

[14] B. Simon and T. Spencer, Trace class perturbations and the absence of absolutely continuous spectrum, Commun. Math. Phys. 125 (1989), 113-126.

[15] G. Stolz, Spectral theory for slowly oscillating potentials, II. Schrödinger operators, Math. Nachrichten (to appear). 